pressure on the lower surface of the hard palate, while ingenious, cannot be regarded as a complete explanation.

It seems very probable that each of these theories is to some extent correct, but that no single one is sufficient to fully explain the mode of production of these deformities. It is, however, quite certain that interference with nasal respiration is capable of producing serious and extensive deformity of the teeth and jaws, and that by the restoration of nasal breathing this disfigurement of the maxilla can be prevented, and if seen at an early stage considerably alleviated, the constant stream of air expanding and developing the upper jaw. There are many cases of V-shaped deformity of the maxilla with anterior protrusion due to other causes, but interference with nasal respiration is so common a cause that the condition of the nose and nasopharynx should be investigated in all these cases.

Breathing exercises as advised by Mr. Arbuthnot Lane are undoubtedly valuable in, some cases of naso-pharyngeal obstruction produced by the presence of an abnormal quantity of adenoid tissue, especially if the obstruction to nasal respiration is a first attack or the repetition of a previous one with a history of free nasal breathing in the interval. But when the obstruction is chronic, especially if acute exacerbations also occur, when secondary aural affections exist, and more especially when the deformities in the teeth and palate above described are present, it will be found that breathing exercises, unless preceded by operative measures, will be almost invariably ineffective, and valuable time will have been irretrievably lost.

REFERENCES

1 Medical Press, igco. 2 Laryngoscope, 1808, 3 Journal Amer. Med. Assoc., igoo. 4 Mouth Breathing, roor.

\section{FOREIGN BODY IN OESOPHAGUS.}

By JOHN McKENZIE, M.A., M.B., CH.B., House-Physician, West London Hospital.

\section{Case.}

J. W., a girl aged 12, was admitted into one of Mr. Paget's wards in the West London Hospital on December $13^{\text {th }}$, 1901 .

History.-She stated that about i p.m., on coming home from school, she seized and swallowed hurriedly a piece of cake which was lying on the counter in her mother's shop. She felt something hard pass over the throat, and found that she was unable to swallow anything more. Neither the child nor her mother having any idea of the nature of the obstruction, various domestic remedies were employed to "get it down." All attempts to swallow pieces of crust, etc., were followed by their prompt return, and about $5 \mathrm{p} . \mathrm{m}$. the child was brought to hospital.

Condition on Examination. - With the $x$ rays a darkcoloured disc about the size of a penny was seen lying, as it were, in the episternal notch, the lower edge of the disc being just behind the upper edge of the manubrium. On looking from the side, a dark line was seen lying almost vertically just in front of the vertebral column. The case was obviously one of a coin impacted in the oesophagus.

Treatment.-All attempts to remove it with coin-catchers and probangs failed, although a small oesophageal bougie was passed into the stomach, and gave a grating sensation on withdrawal. It was decided to attempt its removal under an anaesthetic by means of a laryngeal forceps, and with the help of the $x$ rays. The child was placed on a table lying on her back, and with the vacuum tube close to the left side of the neck. Chloroform was given, and a crocodile-bladed laryngeal forceps was passed into the oesophagus. With the screen placed close to the right side of the neck the blades of the forceps were passed down until they were seen to touch the upper end of the black line; they were then opened slightly and passed down so as to get a firm hold. On putting on light traction the coin refused to move, and I imagined for a moment that the wall of the oesophagus must have got caught in the blades; but with the employment of a considerable amount of force (less has been used in extracting a tooth) the resistance was overcome; and the interesting picture was visible of the coin enclosed in the blades of the forceps moving up towards the month and being finally removed. After the first pull no more force was necessary, and the coin was removed with great ease.. It turned out to be a penny.

After-History.-The patient was discharged from the hospital on the following day. Two days after the operation all that remained was a little discomfort on swallowing.

REMARKS

It appears likely, from all the circumstances of the case, that it would have been impossible to remove the coin by any other means than those employed. Without the $x$ rays it might have been possible to lay hold of the coin, but the force employed was so great that one would have hesitated to employ it without actual demonstration that the coin, and nothing but the coin, was within the blades of the forceps, again, it was so tightly impacted that coin-catchers and probang must have utterly failed to dislodge it, even with the help of the screen.

\section{THE CAUSATION OF DEATH DURING THE} ADMINISTRATION OF CHLOROFORM.

By E. H. EMBLEY, M.D.,

Honorary Anaesthetist to the Melbourne Hospital.

(From the Physiological Laboratory of the University of Melbourne.)

(Continued from page 893.)

III.-The Cause of the Fall of Blood Pressure from the Administration of Chloroform.

ALL previous investigators agree in finding that chloroform causes a gradual fall of blood pressure, but they mostly disagree in their conclusions as to the cause.

Some of these conclusions are summarized in the following scheme :

The Hyderabad Commission maintains that the fall of blood pressure is due to vasomotor paresis alone.

Wood, ${ }^{6}$ Hill, ${ }^{1}$. Hare and Thornton, ${ }^{9}$ MacWilliam, ${ }^{16}$ Duplay and Hallion, ${ }^{17}$ conclude that it is due to vasomotor and heart paralysis combined.

Gaskell and Shore, ${ }^{12}$ Richet, ${ }^{13}$ conclude that the fall of blood pressure is due to impairment of cardiac muscle alone.

The possible. causes o this gradual fall of blood pressure are :

I. Diminution in the heart's output per second.

2. Increase in the outflow from the arterioles, either from: $a$. Loss of central vasomotor control, spinal and medullary.

$b$. Diminished efficacy of the muscular fibres of the arterioles themselves.

3. Increase in the bed of the circulation by relaxation of arteries and veins.

Excepting Gaskell and Shore, the other investigators do not appear to have sufficiently considered the possibility of chloroform directly influencing the muscle tissue in the walls of the vessels themselves, as it does cardiac muscle.

Lister, ${ }^{12}$ experimenting upon the web of a frog's foot, found that the application of chloroform or of chloroform vapour caused hyperaemia of the part. This suggested a local paralytic effect upon the muscle of the arterioles.

I. Changes in the Force of Cardiac Contractions.

It has been shown above that paralysis of the heart occurs from chloroform in the blood eirculating through it, corresponding in vapour tension with small percentages of chloroform vapour in the inspired air. When the circulating blood reaches a vapour tension of chloroform the same as that of 0.3 per cent. vapour in the inspired air, the heart steadily becomes paralysed, and the blood pressure falls.

2. Changes in the Rate of Cardiac Contractions.

That vagus slowing of the heart may be a factor of considerable importance in depressing the circulation is readily seen in those blood-pressure tracings of morphinized dogs in which the percentage strength of chloroform vapour in the air inhaled has been enough to raise the irritability of vagi to the stage of slowing the heart, but not to that of stopping it. 


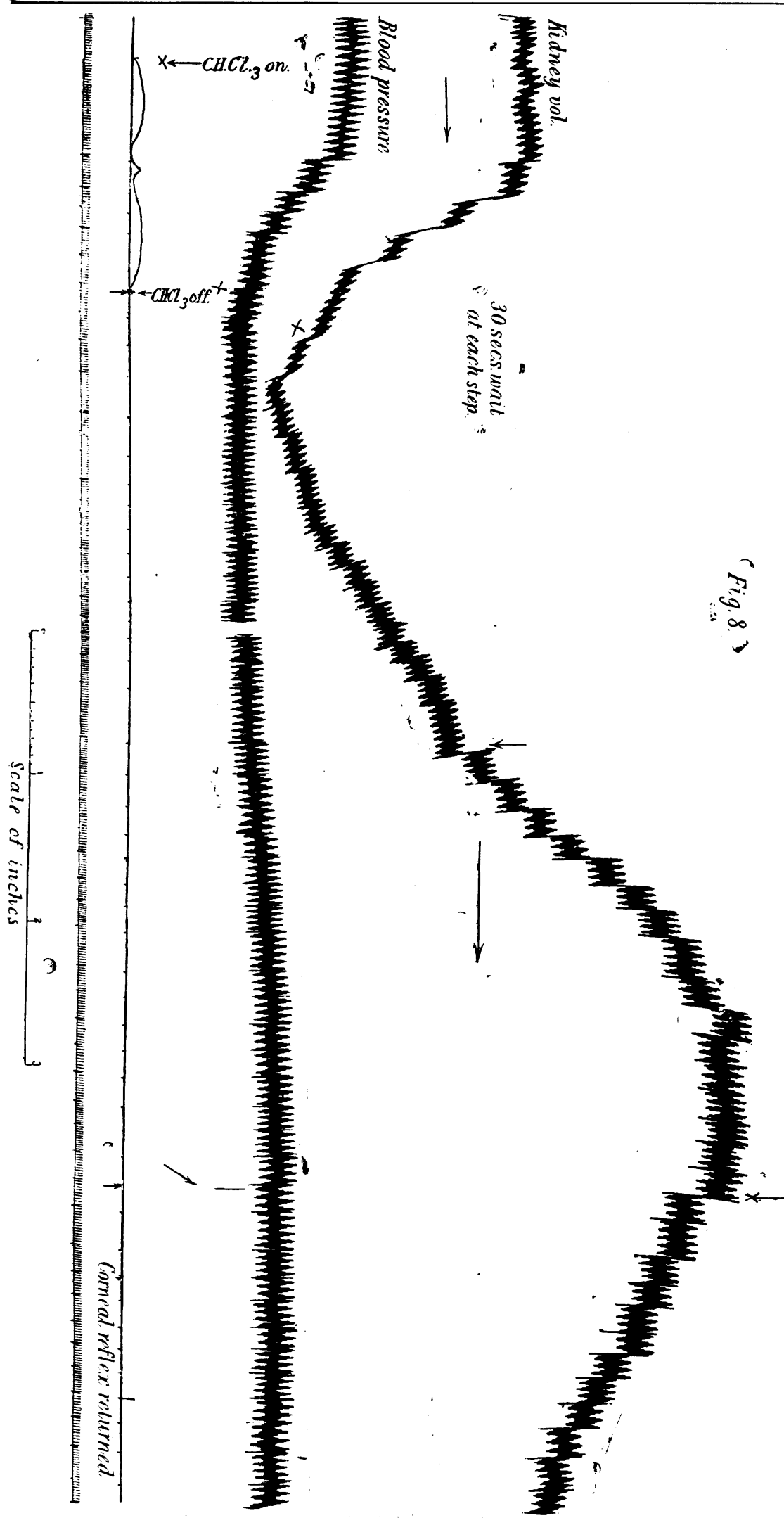

Tracing 2 is an example. In this experiment 2.2 per cent. chloroform vapour was contained in the air inspired by the dog. The heart's rate is seen to slow down from $4 \mathrm{I}$ to 30 per minute, and the blood pressure to fall from 136 to $12 \mathrm{~mm}$. mercury pressure in $25 \mathrm{~min}$. $30 \mathrm{sec}$. The vagi were then divided, whereupon the blood pressure rose steadily to $80 \mathrm{~mm}$. mercury pressure, although the same percentage chloroform was being administered all the time by artificial respiration. The blood pressure does not begin to fall again until 12 min. 5 sec. of further administration, and does not reach zero until 94 min. 30 sec. after the commencement of the experiment.

Two factors are evidently contributing to the fall in blood pressure from this vagal slowing of the heart's rate, namely:

I. The slowing of the heart's contractions and consequent diminution in the heart's output.

2. Further diminution in the force of the heart's contractions consequent upon the increase in the vapour tension of chloroform in the blood to the heart, resulting from the slower passage of blood through the lungs.

The slower the passage of blood through the lungs the more nearly will the vapour tension of the contained chloroform approximate to that of the inspired air.

3. Changes in central vasomotor control." 4. Changes in the efficiency of the muscular walls of the arterioles.

These two questions were investigated by employing simultaneous plethysmographic (bowel, kidney, and spleen) and bloodpressure records of the effects of the inhalation of chloroform of known strength : (a) With brain arteries open; (b) with all brain arteries clamped (including ascending cervical). Similar records were also taken of the effect of injecting blood or saline containing from 0.07 per cent. to 0.7 per cent. by weight of chloroform into the vertebral or internal carotid arteries: (a) With brain arteries open; (b) with brain arteries clamped.

The effects of deepening chloroform narcosis upon the vasomotor response to stimulation of the central end of the sciatic nerve, and upon the vasomotor adjustment to rotation of the animal from the horizontal to the vertical position, were also investigated.

In performing the experiments for obtaining organ volume records, it was found that whereas morphine narcosis was suffi. cient for small operations, curare or ether. anaesthesia was necessary to avoid shock when more extensive operative procedures were required. In obtaining organ volume records, air oncometers were emplozed. These oncometers were made of guttapercha. The kidney and spleen oncometers were of the simple form devised by Schäfer. The bowel oncometer was of the form suggested by Edmonds. ${ }^{25}$ A considerable improvement was made in these oncometers by securing upon the lip of the open part of each oncometer a broad flat ring of brass or glass, ground perfectly true for the adaptation of the glass cover.

Chloroform Administered by Respiration (Brain Arteries Open).

Fifty-nine dogs were experimented upon 


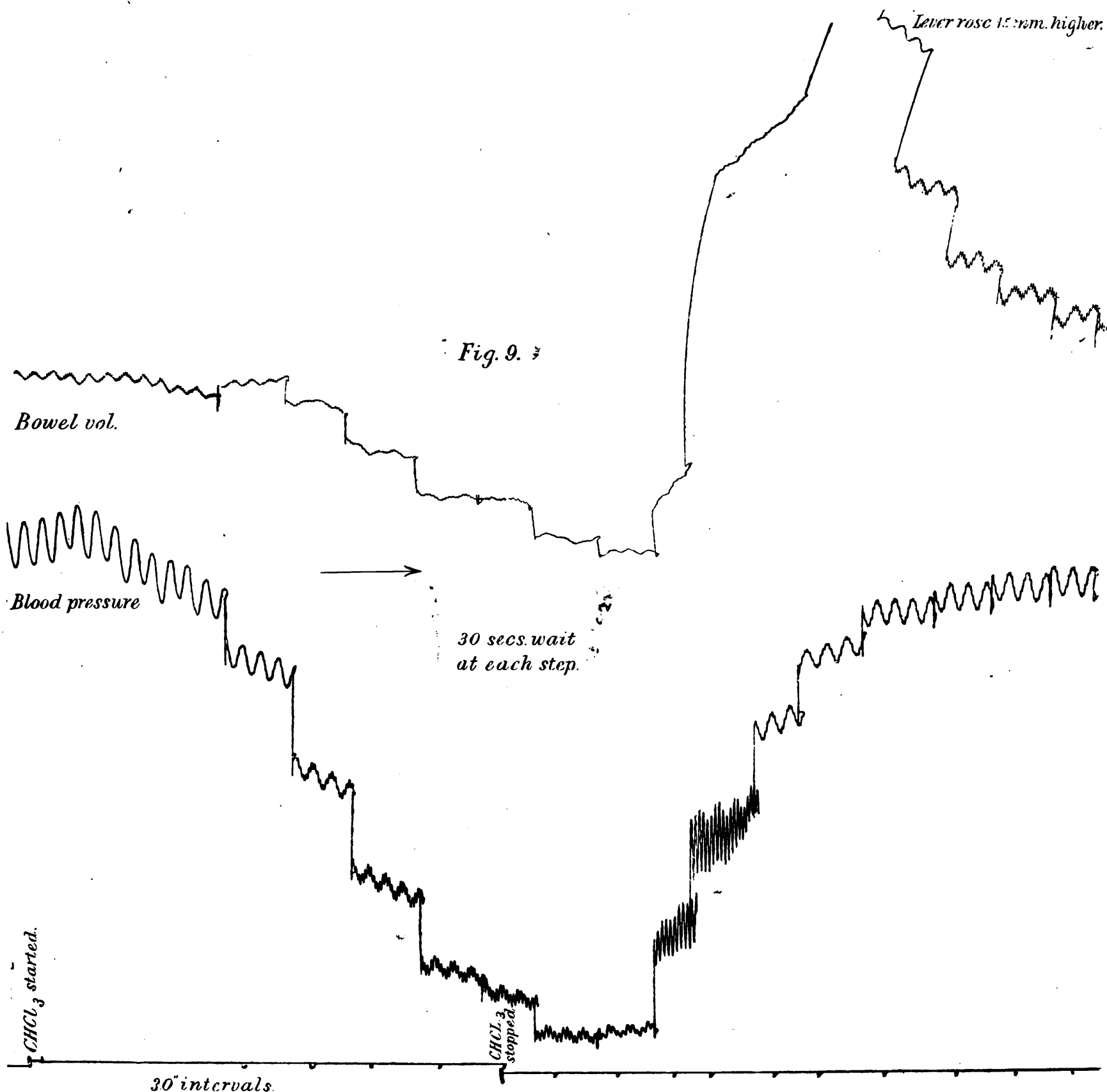

30" intervals.

in obtaining the various organ volume records. The vagi were divided in order to eliminate cardiac inhibition. Artificial respiration was employed.

(a) Kidney Volume Changes.

Twelve animals were used in kidney oncometer experiments. Tracing 8 (p. 952) is representative of the results. It shows that upon the administration of air containing 2.7 per cent. of chloroform vapour, the kidney volume compensated against the falling blood pressure until the fall equalled $14 \mathrm{~mm}$. mercury pressure. As the fall in biood pressure continued the organ volume diminished, so that at the expiration of 3 min. 53 sec., when the administration of . chloroform was stopped, the blood pressure had fallen equal to $40 \mathrm{~mm}$. mercury pressure and the tambour lever had fallen $43 \mathrm{~mm}$.

During recovery a considerable increase occurs in the volume of the kidney, so that at the expiration of $17 \mathrm{~min}$. 12 sec. after cessation of the administration of chloroform, the tambour lever indicates a rise of $80 \mathrm{~mm}$., that is, $37 \mathrm{~mm}$. above the normal, whilst the blood pressure rose equal to $16 \mathrm{~mm}$. mercury pressure only. This increase in the volume of the kidney then began to undergo diminution, so that at the expiration of a further $27 \mathrm{~min}$. 12 sec. it had come down to $7 \mathrm{~mm}$. above the normal. The vessels had recovered. The dilatation of the kidney that occurred during recovery was obviously due to the recovering heart driving blood into the still relaxed. 
vessels and, notwithstanding the low arterial pressure, overfilling them. That this relaxation of the arterioles began to occur early in the course of the administration of the chloroform is to be seen in the compensatory dilatation of the kidney volume that:occurs before the excessive fall of blood pressure obscures it.

(b) Bowel Volume Changes.

Thirty animals were used in measuring bowel volume changes. Tracing 9 (p.953) is representative. It shows that compensating dilatation occurred in the bowel vessels for the fall of blood pressure, from the administration of air containing 25 per cent. chloroform vapour. The compensation is more complete than was the case with the kidney. The relaxation of the bowel vessels compensated against the fall in blood pressure for 72 sec. During this period the blood pressure had fallen equal to $40 \mathrm{~mm}$. mercury pressure. The bowel

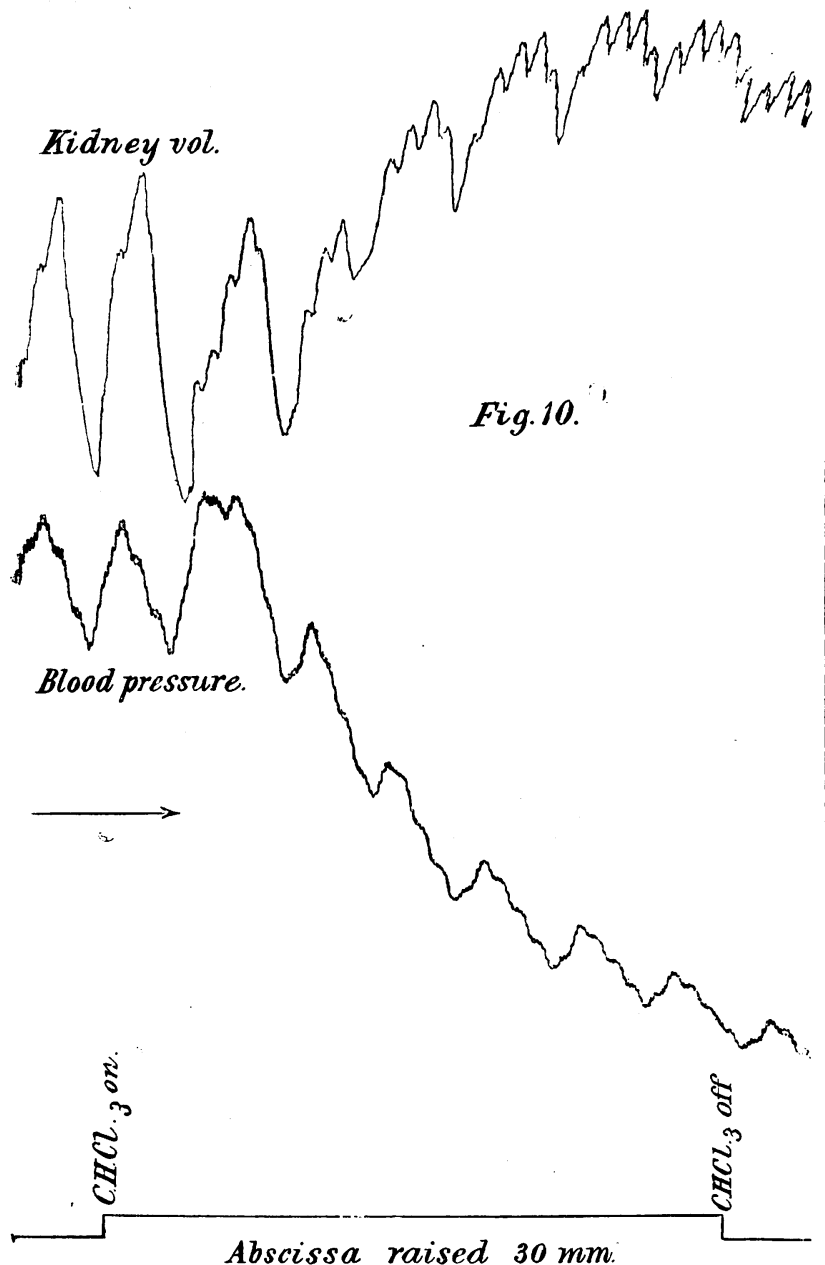

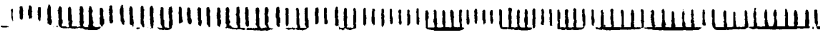
volume then began to diminish. The diminution, however, was not great, as the lever of the tambour indicated a fall of only $28 \mathrm{~mm}$., whilst the fall of blood pressure was equal to $56 \mathrm{~mm}$. mercury pressure at the time when the administration of chloroform ceased. The recovery, on the other hand was attended by a greater increase in volume than that which occurred in the case of the kidney. The lever indicated a rise of $80 \mathrm{~mm}$. above the level of normal volume at the expiration of 2 min. $44 \mathrm{sec}$ after the administration of chloroform had ceased. During this time the blood pressure had recovered to within $58 \mathrm{~mm}$. mercury pressure of its normal level. As the blood pressure continued to rise the bowel volume began to contract, so tinat the lever of the tambour fell slowly as the vessels were recovering their tone. (c) Spleen Volume Changes.

Seven experiments were made with the inhalation of varying percentages of chloroform in air, during which simultaneous records of the blood pressure and volume of the spleen were taken. The results corresponded with those obtained with the bowel and kidney. The rhythmical contractions of the organ diminished and ultimately ceased as the anaesthesia progressed.

The records show that upon discontinuing the administration of chloroform in each instance the heart recovers before the vessels. The organs at first always continued to increase in volume as the blood pressure rose. The period during which dilatation of the organ continued to occur before the onset of constriction or recovery varied with the duration of the administration. If the administration had been continued for 20 or 30 sec. only, the recovery in blood pressure and organ volume was almost simultaneous. If, on the other hand, the administration was continued for $4 \mathrm{~min}$. the vessels did not begin to constrict until 17 min. 12 sec. after administration had ceased. Meanwhile the heart had recovered, and had been distending the vessels with blood.

(b) Brain Arteries Clamped.

This was accomplished by clamping the carotids, the vertebrals, the ascending cervical arteries, and the thyroid axes. The four jugular veins were also tied and the vagi were cut. The animals were curarized, and artificial respiration was performed throughout.

In these experiments chloroform was largely excluded from the brain. A small amount of chloroform reaches the brain by way of the foramen magnum. Changes occurring in the organ volumes are less complicated by the influences of chloroform on the medulla.

When chloroform was administered under these conditions the fall of blood pressure was always accompanied by a simultaneous increase in organ volume, indicating even more marked relaxation of the arterioles.

(a) Kidney Volume Changes.

Five animals were employed in obtaining simultaneous tracings of blood pressure and kidney volume.

Tracing ro is typical. It shows that at the expiration of 25 sec. after the administration of air containing 3.5 per cent. of chloroform vapour, the organ volume had increased so that the lever of the tambour indicated a rise of $7 \mathrm{~mm}$., whilst the blood pressure had fallen $50 \mathrm{~mm}$. of mercury pressure; that at the expiration of 62 sec., at which time the administration of chloroform vapour was stopped, the lever indicated a rise of $15 \mathrm{~mm}$., whilst the blood pressure had fallen $98 \mathrm{~mm}$. of mercury pressure. The volume then began to diminish and to rise again with the recovery of the heart and blood pressure, so that at the expiration of $44 \mathrm{sec}$ after the administration had ceased the tambour lever was $5 \mathrm{~mm}$. above its normal and the blood pressure had risen $6 \mathrm{~mm}$. mercury pressure.

In this tracing the Traube-Hering curves are more than usually marked.

(b) Bowel Volume Changes.

Five animals were employed in obtaining this class of record, of which Tracing I ( p. 955) is typical. It is the result of administering air containing 2.5 per cent. of chloroform vapour. The increase in volume of the bowel occurred simultaneously with the fall in blood pressure, and corresponds with that which occurred in the experiments with the kidney volume. It shows that in 42 sec. of administration the increase in bowel volume had raised the lever of the tambour so that it indicated a rise of $6 \mathrm{~mm}$., whilst the blood pressure had fallen $20 \mathrm{~mm}$. mercury pressure; that in 94 sec., when the administration of chloroform was stopped, the lever indicated a rise of $16 \mathrm{~mm}$., while the blood pressure fell equal to $70 \mathrm{~mm}$. mercury pressure; that in 35 sec. after the administration of chloroform had ceased, the lever indicated a rise to $19 \mathrm{~mm}$., while the blood pressure fell another ro $\mathrm{mm}$. mercury pressure. The blood pressure than began to recover, but the bowel volume continued to increase for 42 sec. more before it began to fall; then the blood pressure began to rise more rapidly, so that $66 \mathrm{sec}$. later the lever had fallen $12 \mathrm{~mm}$., whilst the blood pressure had risen $12 \mathrm{~mm}$. mercury pressure.

The results obtained indicate a decided increase in organ 


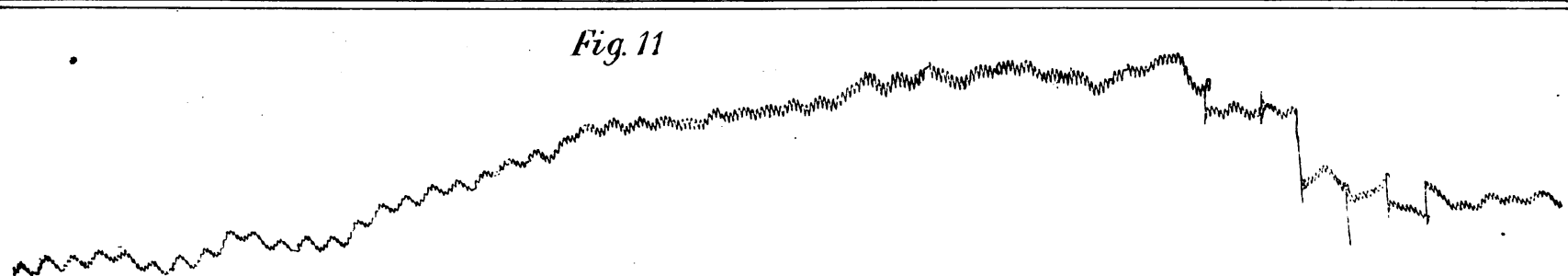

Bowel vol.

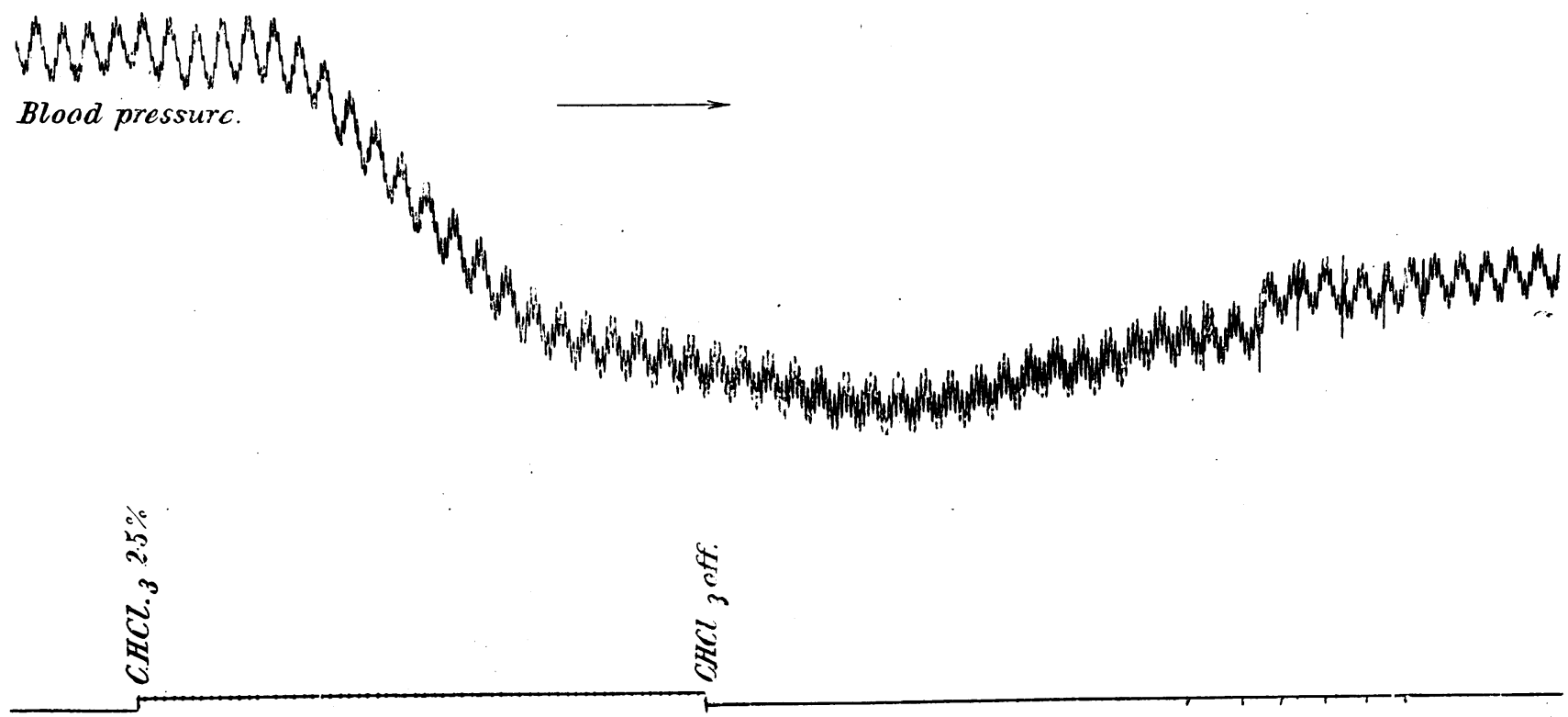

Waits $15 \sec 3$. cash.

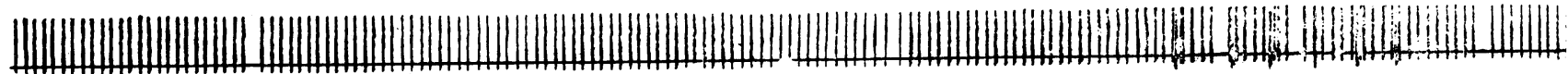

volume during a great fall in blood pressure. The increase in organ volume was distinctly more than occurred when the brain arteries were open. As this greater dilatation occurred when the chloroform was practically excluded from the brain, it is obvious that the dilatation is not due to any medullary vasomotor paralysis. It must therefore be due to paralysis of the spinal vasomotor centres or to paralysis of the muscle cells in the walls of the arterioles.

Further experiments were then performed with a view to elucidating the matter further, in which chloroform was delivered directly to the brain by injection into the vertebral or internal carotid arteries.

In these experiments the direct effects of chloroform on the vasomotor centres in the medulla were studied. The object aimed at was to deliver chloroform to the brain alone and observe the effects produced upon the vasomotor centres in the medulla uncomplicated by any heart or peripheral vascular effects.

Gaskell and Shore ${ }^{12}$ performed a number of this class of experiment. They injected undiluted chloroform into the internal carotid or vertebral arteries. They obtained a decided rise of blood pressure. These results, together with those of their cross circulation experiments, led them to conclude that chloroform inhalation did not paralyse the vasomotor centres in the medulla.
Hill, who concluded from his experiments in vertical rotation of animals that the vasomotor system was paralysed by the inhalation of chloroform, inferred that the injection results of Gaskell and Shore were produced by anaemia of the bulb. He advances, in support of this contention, that nitrite of amyl, in the hands of Cash and Dunstan, produced the same result as chloroform when injected into the carotid artery. This, however, by no means supports his contention. I find, with Cash and Dunstan, that nitrite of amyl actively stimulates the vasomotor central systemfor example, $4 \mathrm{c.cm}$. of I per cent. in normal saline at $40^{\circ} \mathrm{C}$. injected into the internal carotid artery caused a rise of blood pressure equal to $100 \mathrm{~mm}$. mercury pressure; that, on the other hand, when the brain arteries were clamped and the nitrite was given by inhalation, there was a great and prompt fall of blood pressure accompanied by very marked peripheral dilatation of the vascular system, as shown by oncometer measurements of the kidney and bowel. Its effect upon the vascular system is, in fact, the same as that of chloroform, but much more rapidly paralytic upon the peripheral vessels. This accords with the conclusions of Brunton ${ }^{2 \triangleleft}$ in respect to nitrite of amyl.

In order to avoid the possibility of the bulbar anaemia charge being advanced to account for the results of these experiments, very weak solutions of chloroform in normal saline 
or defibrinated blood were for the most part used. Controls were also made by injecting normal saline solution or defibrinated blood alone. In no instance were such control injections found to produce any effect upon the blood pressure.

The lowest percentage strength of chloroform used was 0.075 per cent. by weight. Injections of 20 to $40 \mathrm{c.cm}$. were employed. Further dilution to an uncertain extent by the cerebral blood took place. Such diluted chloroform produced a decided rise of blood pressure, with simultaneous constriction of organ volume, both of which passed off with equal rapidity.

Solutions of chloroform in defibrinated blood could not safely be made stronger than 0.15 per cent. Higher percentages had the effect of laking the blood and causing masses of haemoglobin crystals to occur.
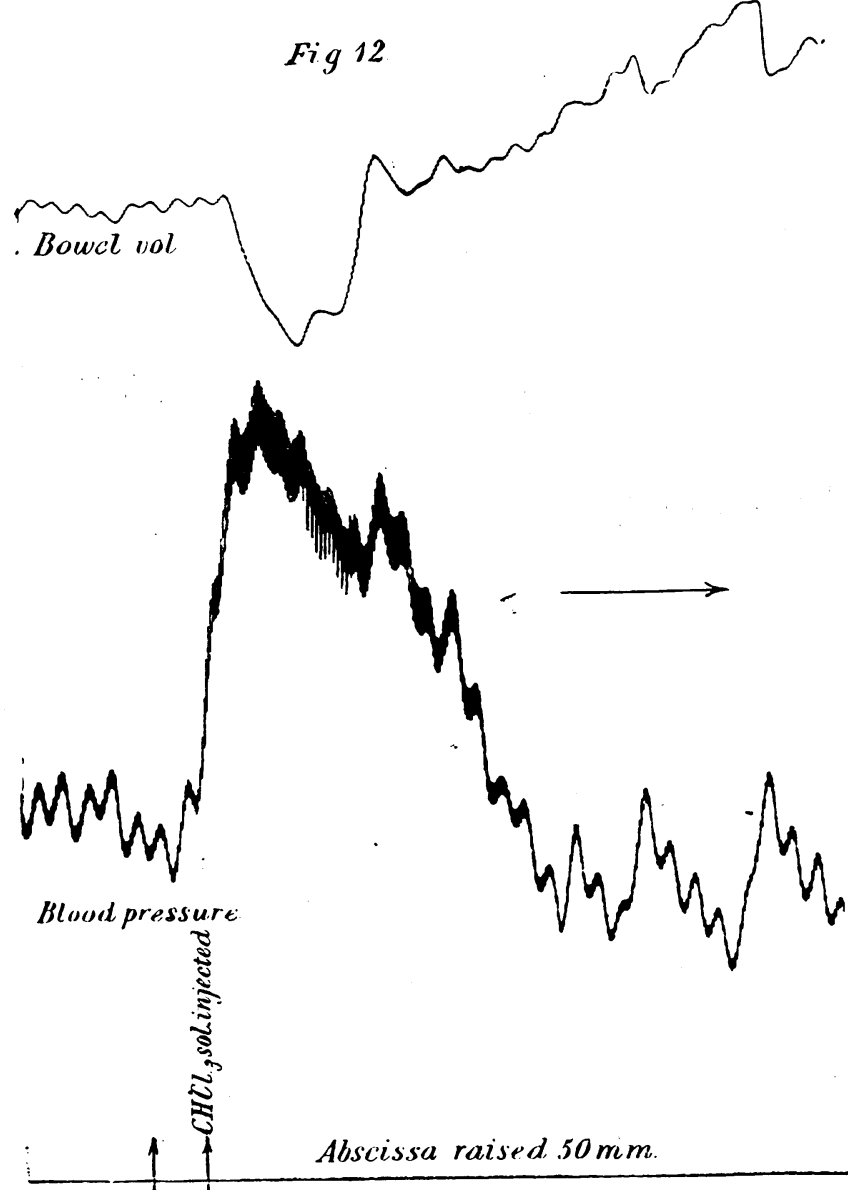

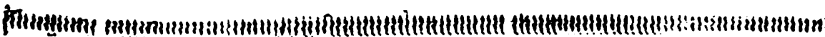

In most of these experiments the carotid and vertebral arteries were clamped and the vagi were cut. The internal carotid artery or arteries were connected with a pressure bottle containing the chloroform solution maintained at $40^{\circ} \mathrm{C}$. by a water bath. When the blood pressure had become clamp of the brain arteries, the chloroform solution was injected at approximately the systemic blood pressure. In a few of the experiments the injections were made into the vertebrals. The same result was obtained.

The moderate amount of arterial blood that finds its way to the medulla by way of the foramen magnum in no way impairs the results of this class of experiment. The same experimental results are obtained, if, in addition to clamping the four arteries mentioned, the thyroid axis and the ascend- ing cervical arteries be tied and the aorta clamped just beyond the subclavian artery. By these measures the circulation is carried on by the brachial arteries only and the blood supply is cut off from the brain. The blood pressure promptly rose upon the injection of chloroform under these conditions as readily as when the injections were made with the aorta and small arteries open.

The small amount of blood that reaches the medulla through the spinal canal is an advantage on the other hand, for it prevents actual asphyxiation of the vasomotor centres during the necessary operative procedure.

The remarkably free venous anastomosis along the spinal canal is important, however, since by its means chloroform which has passed through the cerebral circulation is easily carried into the general circulation, though the four jugular veins are tied.

Tracing 12 illustrates the effect of injecting $20 \mathrm{c} . \mathrm{cm}$. of 0.7 per cent. chloroform in normal saline solution at $40^{\circ} \mathrm{C}$. The injection of this amount extended over $8 \mathrm{sec}$. It produced a rise of blood pressure equal to $90 \mathrm{~mm}$. mercury pressure. Simultaneously with this rise in pressure the bowel volume constricted so that the lever of the oncometer tambour fell $15 \mathrm{~mm}$.

In an experiment with 0.3 per cent. solution of chloroform in normal saline, $25 \mathrm{c.cm}$. were injected, it caused a rise of $80 \mathrm{~mm}$. mercury pressure and a fall of $18 \mathrm{~mm}$. in the tambour lever.

An experiment in which $40 \mathrm{c.cm}$. of 0.075 per cent. solution of chloroform in defibrinated blood was injected, a rise of blood pressure equal to $50 \mathrm{~mm}$. mercury pressure resulted.

These experiments were performed upon 36 animals. A rise of blood pressure occurred in every one.

The response to the stimulus of chloroform injection into the brain by the vasomotor centres in the medulla is not one occurring only in the initial stage of the administration. I have found it to occur upon the injection of 0.7 per cent. chloroform solution after 103 min. of sound surgical anaesthesia, the brain arteries being open meanwhile. The effect of the injections at the expiration of I03 min. was, of course, much less marked than it was at the commencement. The vasomotor system was then by no means paralysed, as the blood pressure was still equal to $84 \mathrm{~mm}$. mercury pressure. Since the effect of chloroform upon the vasomotor centre in the medulla is stimulative for a considerable time, whereas the actual effect upon the vessels is relaxation, it would appear that the paralytic effect is due to the action of chloroform upon the muscle cells or spinal centres.

\section{Faradizing:the Sciatic Nerve under Deepening Chloroform} Narcosis.

The reflex vasomotor effects produced by faradization of the sciatic nerve showed progressive failure as the chloroform narcosis deepened.

Faradizing the sciatic nerve of a curarized dog after 7 min. 5 sec. of the administration of air containing 2.4 per cent. chloroform vapour, caused a rise of blood pressure equal to $30 \mathrm{~mm}$. mercury pressure. After a period of 9 min. 30 sec. administration, the same stimulus caused 9 min. 30 sec. stration was now stopped for $5 \mathrm{~min}$. 30 sec. Air containing 3.5 per cent. chloroform vapour was then administered, and 3.5 per cent. chloc faradization of the sciatic nerve caused a rise of blood pressure equal to $14 \mathrm{~mm}$. mercury, and after $3 \mathrm{~min}$. 1o sec. a rise equal to $9 \mathrm{~mm}$. mercury pressure. The 3 ministration was then stopped, and after 2 min. 10 sec. faradizing caused a rise of blood pressure equal to $12 \mathrm{~mm}$. mercury pressure.

\section{Experiments upon the Effects of Vertical Rotation under} Chloroform.

The results of vertical rotation experiments corroborated those of Hill.1 There does not appear to me, however, to be any grounds for necessarily assuming central vasomotor paralysis from these rotation experiments. Paralysis of the muscular tissue of the arterioles would equally account for the failure of adjustment on rotation, such as occurs in animat not chloroformed. Another factor was found in these experiments which Hill does not appear to have observed, 


|
作 that aids in depressing the circula-

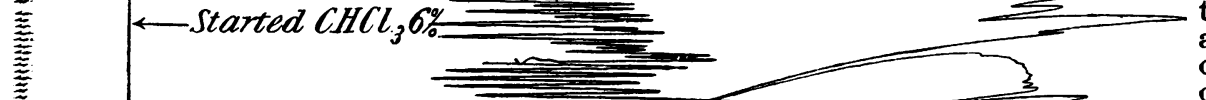
animal, namely, that the heart's rate does not, increase on rotation as it does when the animal is not narcotized by chloroform. The increased rate that occurs upon rotating a dog merely morphinized helps by increased output to sustain the blood pressure. When the animal is being chloroformed this compensatory factor is rapidly lost, as the chloroformization deepens. This may be due to the effect of chloroform in increasing vagal irritability, but a complex reflex adjustment will naturally become less adequate and ultimately abolished under the influence of chloroform.

Conclusions.

I. Chloroform causes a diminution of vascular tone of the arterioles.

2. Diminution in the vascular tone of the arterioles is most obvious when chloroform is practically kept out of the brain

3. The central vasomotor system is stimulated, at any rate for a time, by chloroform.

4. The cause of the fall of blood pressure from the administration of chloroform is paralysis of the muscle cells of the heart and of the arterioles (provisional). The fall may be further augmented by slowing of the heart's rate, or suddenly brought to zero, by vagus inhibition of the heart.

IV.-The Relation of Asphyxia To Syncope and THe Causes of RESPIRATORY FAILURE.

Analysis of 38 blood-pressure and respiration tracings which were obtained in investigating this section shows that fall of blood pressure contributes to respiratory failure. Chloroform may paralyse the respiratory nervous mechanism independently of a fall in blood pressure but such results have never occurred in any of my experiments in which the chloroform was administered by inhalation. Analysis showed that in 31 out of $3^{8}$ experiments the blood pressure was below $50 \mathrm{~mm}$. of mercury pressure before respiration failed. Of the 6 other experiments the highest blood pressure was 7o $\mathrm{mm}$. mercury pressure when the respiration ceased. Most of the failures of respiration occurred when the blood pressure was about $44 \mathrm{~mm}$. mercury pressure.

The part which fall of blood pressure plays in conducing to respiratory failure is illustrated in Tracing I4 (p. 958). In this experiment the dog was not morphinized preliminarily. The connexions for the manometer and the respiration were prepared with the dog under ether, ei yht hours previously. It shows that respiration failed 15 sec. after blood frassure had, from vagus inhibition of the heart, reached zero. Further, it shows that after 90 sec., the vagus mechanism failed longer to inhibit the heart, and the blood pressure rose. 


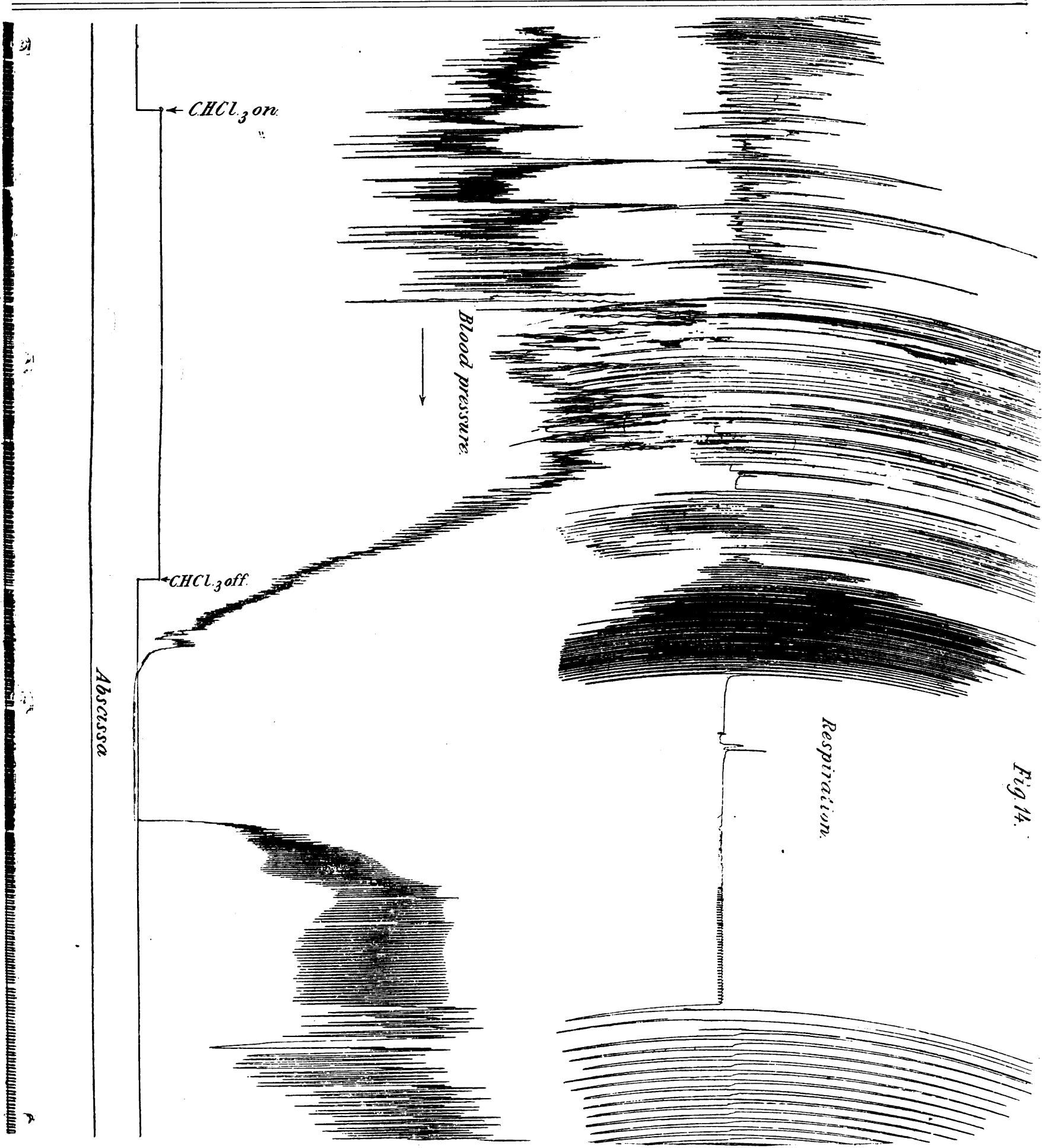

When the blood pressure reached $100 \mathrm{~mm}$. mercury pressure the respiration returned.

Tracing 13 (p. 957) further illustrates the effect of fall of blood pressure in conducing to respiratory failure. It is another case of vagus inhibition of the heart. In this experiment, however, the vagi were divided at the position indicated, in order to raise the blood pressure. The respiration had failed when the blood pressure was at $16 \mathrm{~mm}$., and returned when the blood pressure had risen to $92 \mathrm{~mm}$. mercury pressure. Chloroform was inhaled so long as the animal breathed.

Further confirmaticn was obtained by performing rotation experiments. The results so obtained corroborated those of Hill. ${ }^{1}$ Respiration was found to fail when the blood pressure of a chloroformed dog fell upon rotating the animal vertically into the head-up position, and to return when the blood 
pressure rose after rotating the animal back into the horizontal position.

Explanation OF TRACINGS.

Tracing 8.-(Organ valume measurements.) Oncometer and bloodpressure tracing. Upper tracing is kidney volume, the lowe tracing is blood pressure. Brain arteries open. Vagi cut. Dog weight $16 \mathrm{lb}$. Morphine 0.25 gram, curare o or6 gram. Artificia respiration. Chloroform vapour 2.7 per cent. in the air administered: Administration continued for 3 min. 55 sec., as indicated by the bracket sign. Drum was stopped 20 times for 30 sec. each, 15 times for $x$ min. each-that is, between the first and second arrows tand

and

Tracing 9.-(Organ volume measurements) Brain arteries open. Bowel oncometer (B. V.) and blood-pressure (B. P.) tracings. Dog, $20 \mathrm{lb}$. Morphine, o.3 gram. Curare, o.o2 gram. Vagi cut. Chloro form vapour, 2.4 per cent in the air administered by artificial respiration during the period of elevation of the zero line. Recording drum was stopped 20 times for periods of 30 sec. each. The lever of the tambour rose ${ }_{5} \mathrm{~mm}$. over the drum during the heart's recovery.

Tracing ro.-(Organ volume measurements with brain arteries clamped.) Kidney volume (K. V) and blood-pressure (B. P.) tracings. Botl Kidney volume (K. Y) and blood-pressure (B. P.) tracings. Botl carotids and vertebrals and the other ascending arteries clamped and the vagi cut. Chloroform 3.5 per cent. vapour in the ais administered by artificial respiration during the period of eleva tion of the zero line.

Tracing i r.-(Organ volume measurements with brain arteries clamped.) Bowel volume (B. V ) and blood-pressure (B. P) tracing. Bowel volume (B. V.) and blood-pressure (B. P.) tracing. Brain arteries clamped and vagi cut, as in ro. Morphine, o.4 gram Curare, 0.02 gram. Chloroform, 2.5 per cent. vapour in the air by elevation of zero line. Six times the drum was stopped, by elevation of zero line.

Tracing r2.-(Effect of chloroform delivered to brain.) Bowel volume (B. V.) and blood pressure (B. P.) tracings. Dog, weight $25 \mathrm{lb}$ Morphine, 0.4 gram. Curare, o.025 gram. Vagi cut. Artificial form in saline solution at $40^{\circ} \mathrm{C}$. into the internal carotid artery, during the interval of time between the arrow heads. Time line is zero line.

Tracing 13.-(Relation of respiration to circulation.) Respiration the upper, and blood pressure the lower, tracing. Dog, weight $33 \mathrm{lb}$. Morphine 0.5 . Chloroform 6 per cent. in respiration at "started" and continued till the respiration ceased. Respiration ceased when the blood pressure was equal to r $6 \mathrm{~mm}$. mercury pressure. Vagi were cut when the blood pressure rose. Respiration recommenced when the blood pressnre had reached $92 \mathrm{~mm}$. mercury pressure.

Tracing 14. (Relations of respiration and circulation.) Respiration upper, blood pressure lower, tracings. Non-morphinized dog. Tracings include the induction period. Dog. weight $24 \mathrm{lb}$. Chloroform given from a towel during a period indicated between arrow heads marked "started" and "off." Much struggling during early part of administration. Respiration ceased $16 \mathrm{sec}$ after blood pressure had fallen to zero by vagus inhibition of the heart. Respiration recommenced when the heart, having recommenced, had raised the blood pressure to equal $102 \mathrm{~mm}$. mercury pressure.

Spontaneous Recovery of Respiration.

In 8 experiments out of the 38 the respiration recovered spontaneously. In 6 of these spontaneous recoveries vagus inhibition of the heart had dangerously lowered the blood pressure before respiration ceased.

The blood pressure in these eight experiments was : $\circ$ when respiration failed, and $60 \mathrm{~mm}$. Hg. when it returned.

$$
\begin{aligned}
& \text { o " } 72 \text { " Hg. when it } \\
& \begin{array}{rrrr}
\circ & , & \text { 102 } & , \\
34 & , & 60 & ,
\end{array} \\
& \begin{array}{rrrr}
16 & , & 92 & ,
\end{array}
\end{aligned}
$$

Continuance of "respiration under "chloroform"anaesthesia therefore, greatly depends upon the maintenance of the blood pressure. This result is in accord with the conclusions of Gaskell and Shore, and Hill.

In 24 of the 30 cases in which respiration did not recover the combined stimulus of chloroform and asphyxia occasioned permanent inhibition.

Asphyxia, as is well known, greatly increases the irritability of the vagus centres under cllloroform. So much so is this the case that when the vagus mechanism has become irresponsive to even 6 or 7 per cent. chloroform vapour in the air administered, the additional stimulation of asphyxia from arrest of respiration often raises the vagus irritability to the extent of arresting the heart in morphinized dogs.

Conclusions in Respect to the Relations of Respiration and

Circulation in Dogs, and the Cause of Respiratory Failure.

I. Failure of respiration is mainly due to fall in blood pres sure. With a good blood pressure, failure of respiration by inhalation of chloroform is practically impossible.

2. Restoration of failed respiration is dependent upon restoration of blood pressure.

3. Failure of respiration occurring in the induction or early period of anaesthesia happens as frequently after as it does before the heart stops.

\section{V.-The Effect of Varying Percentages of Chioroform}

Vapour in the Air Breathed UPON THE Results.

Snow, ${ }^{3}$ Paul Bert, ${ }^{22}$ Dubois, ${ }^{23}$ and more recently Waller, ${ }^{15}$ have by various methods of investigation determined that I per cent. to 1.5 per cent. (by volume) chloroform vapour in the air breathed may be regarded as the anaesthetic range for chloroform, and that percentages above 1.5 per cent. become lethal. In my own experiments these figures represent an approximate guide for fairly continuous administration.

Considerable difference of time occurs before anaesthesia is attained in different animals, even when using the same percentage chloroform vapour in the air inspired. Old dogs, for instance, take a much longer time to become anaesthetized than do young dogs of the same weight. Similarly, and from obvious physiological reasons, big dogs take longer to anaesthetize than little ones. The difference in the time taken to anaesthetize a big and old dog on the one hand and a small puppy on the other with the same percentage chloroform is very great.

The results, moreover, show that there is no actual line between anaesthetic and lethal percentages. It is a question of time only for percentages lower than the anaesthetic ones to kill by continuous inhalation. Dastre ${ }^{10}$ has already pointed this out.

The question of what is the fatal saturation point in the whole body never occurs in practical anaesthesia. In fact, during inhalation the venous blood probably never, under any conditions of practical administration, reaches the same vapour tension of chloroform as the arterial.

The consideration of the effects of varying percentages of chlcroform vapour in the air inspired has a practical bearing, and that is in its relation to the occurrence of sudden deaths under chloroform. The vapour tension of chloroform in the arteries therefore is the question involved. This is so because the tissues of the body are for a considerable time the receptacle into which the arterial blood discharges most of its charge of chloroform.

Tracing I demonstrates the effects of arrest of distribution of chloroform, and therefore the rapidity with which the vapour tension of chloroform ( 1.2 per cent.) in this small amount of circulating blood attains that of the air inspired. $\mathrm{By}$ the experimental method in which this tracing was obtained, the circulating blood passes either by one carotid artery through a short artificial circuit to the corresponding external jugular vein, or through the coronary circuit. By this means the disposal of chloroform by the arteries is arrested and the circulating blood rapidly obtains the same vapour tension of chloroform as that of the inspired air. In this experiment with 1.2 per cent. (anaesthetic percentage of Paul Bert ${ }^{22}$ and others) chloroform vapour in the air, the heart was paralysed in 9 min. 45 sec. of administration.

By the same method the effect of other percentages was ascertained. The results of three experiments with different percentages are here given, and are placed in contrast with the effects of nearly similar percentages administered with the whole circulation of the animal open. The anaesthetic was administered hv artificial respiration in each series.

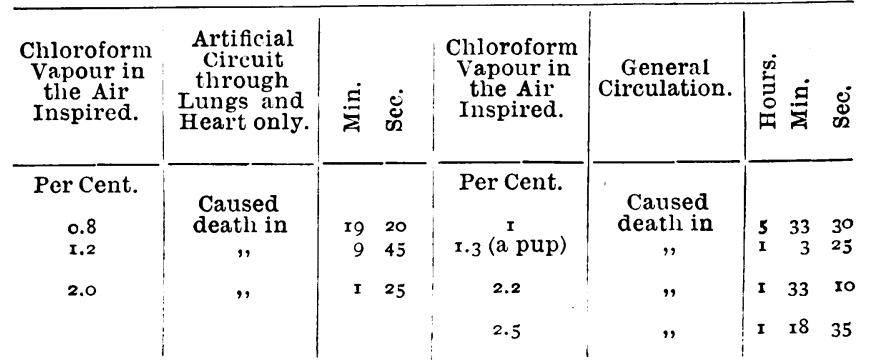


In the experiments with 2.2 per cent. and 2.5 per cent. chloroform vapour in the air inspired and the general circulation open, inhibition of the heart occurred at the expiration of 15 and $24 \mathrm{~min}$. respectively, so that vagotomy had to be performed.

With the small quantity of blood in this limited circulation the effect is very rapid, and as seen in Tracing 2 recovery is correspondingly quick. The contrast indicates that blood in the lungs does not rapid]y acquire the same vapour tension as the air breathed, in ordinary chloroformization, by once traversing the pulmonary capillaries. In normal conditions it parts with much of what it has taken up in the lungs, to the fymph and tissues of the general circulation, so that a considerable time, depending upon the size of the animal, must ensue before equilibrium between air and blood obtains. With the limited circulation and small quantity of blood on the contrary, this equilibrium occurs within a few minutes.

The relative excitability of the vagus mechanism to the stimulation of chloroform appears, however, to be an important varying factor in all questions of percentages. It has been found that 2 per cent. chloroform vapour in the air inspired is liable to bring on fatal vagus inhibition in the dog.

Concrusions.

I. The blood of the lungs, by once traversing the pulmonary capillaries, does not attain to nearly the same vapour tension of chloroform as that of the air inspired.

2. The vapour tension of chloroform in the arteries is the important practical question. As this rises and falls with the volume of chloroformed air inspired, also with variation in vapour tension of the chloroform in the air, it follows that the effects of chloroform upon the animal will vary with the depth and rate of the respirations, just as much as with the percentage strength of chloroform vapour in the air administered.

3. Chloroform in arterial blood is largely discharged into the tissues at first-that is, the vapour tension of chloroform in the venous blood is equal to that of the tissues. As the arteries continue to pour more chloroform in, the vapour tension of chloroform in the tissues and venous blood would gradually approximate to that of the arteries.

4. The range of percentage of chloroform vapour in the air inspired, for inducing chloroform anaesthesia in the same period of time in different dogs, varies between 0.8 per cent. and 2.5 per cent.

5. Two per cent. or upwards of chloroform vapour in the air inhaled is liable to bring on dangerous vagus inhibition.

\section{VI.-Summary of Concrusions and Brief Discussion of} Results.

1. Heart muscle is very sensitive to the poisonous effects of chloroform.

These conclusions are in accord with those of Ringer ${ }^{4}$ Wood, ${ }^{6}$ MacWilliam, ${ }^{7}$ Hare and Thornton, ${ }^{9}$ Gaskell and Shore, ${ }^{12}$ Richet $^{13}$ and Hill. ${ }^{1}$ 'They emphatically refute the assumption of the Hyderabad Commission ${ }^{8}$ and Lawrie ${ }^{27}$ that chloroform by inhalation never impairs the heart.

2. Chloroform raises the excitability of the vagus mechanism particularly in the early part of the administration.

This has been recognised by the French investigators Morat and Doyen ${ }^{11}$ and Dastre ${ }^{10}$ also by MacWilliam ${ }^{7}$ and Brodie and Russell.17

The increased excitability of the vagus mechanism is due to the action of chloroform on the centres.

This is in agreement with the conclusions of Morat and Doyen $^{11}$ and MacWilliam. ${ }^{7}$ Dastre ${ }^{19}$ however, attributes it to reflexes arising from stimulation by chloroform of the sensory terminals, particularly, of the nasal and snperior laryngea nerves along the respiratory tract. Brodie and Russell ${ }^{17}$ attribute it to the same cause as Dastre, but they emphasize, in addition, the vagus terminals in the pulmonary alveoli as the cause of the vagus effects. Reflexes such as Dastre and Brodie and Russell obtained, do occur, but I have, I think demonstrated above that the fatal inhibitions which occur when the blood pressure has fallen considerably are not so produced.

The inhibitory action of the vagus is more intense and fatal in its effects from being exercised upon a heart whose spon. taneous excitability is diminished by the action of chloroform upon it.
Chloroform vapour, not stronger than 1.5 per cent., in the air administered to morphinized dogs, after a period of mild excitation, slowly depresses vagus excitability. Above 2 per cent. in the air inhaled, may occasion dangerous or persistent inhibitions in dogs.

The failure of many experimenters to recognise the importance of vagus inhibition of the heart appears to depend upon the fact that the inhibitory mechanism was paralysed or exhausted by the previous induction of chloroform anaesthesia before the records of the experiments were commenced This especially applies to the Hyderabad Commission ${ }^{8}$ and Richet. ${ }^{13}$ A dog becomes, in fact, quite another animal after the induction of chloroform anaesthesia without morphine; from being an animal with a very sensitive vagus control of the heart, he becomes one almost wholly devoid of this function, that is, if he lives through the induction. Consequently vagus inhibition from chloroform alone, or from chloroform and asphyxia combined, or from reflexes arising from surgical procedures, are difficult to demonstrate in dogs so prepared.

Similarly the inferences of the Hyderabad Commission with respect to the time relations of cardiac and respiratory failure, although true for their experiments, do not necessarily apply for animals in which the vagus mechanism, the cause of sudden circulatory failure, is not exhausted. They did not take any records of the induction period of chloroform anaesthesia, the period in which the vagi are active. ${ }^{8}$

The fall of blood pressure has been shown to be largely due to the direct effect of chloroform upon the heart. Heart failure begins with the fall of blood pressure. All blood-pressure tracings, those of the Hyderabad Commission as well, show that this precedes failure of respiration.

Richet, ${ }^{13}$ on the other hand, and curiously enough from results obtained from experiments performed upon animals with similarly exhausted vagi, concluded that clinical deaths never result from failure of respiration. He found that after failure of respiration the circulation continued from 3 to 5 mins. Richet ${ }^{13}$ evidently did not obtain even the feeble vagus effects that the Hyderabad Commission got.

The recovery of vagus function after the depression resulting from the induction of chloroform anaesthesia, is slow, and I wish to emphasize the fact that transient recovery followed by reinduction does not represent the conditions of a primary induction.

Section of the vagi or atropinization of dogs absolutely abolishes sudden heart arrest from chloroform. The heart under such conditions fails slowly with rapid pulse, slowing as the blood pressure approaches zero. Under these conditions the respiration fails when the blood pressure falls sufficiently, and invariably long before the heart stops.

This conclusion coincides with that of Dastre, ${ }^{10}$ Morat and Doyen, ${ }^{11}$ but not with that of Richet. ${ }^{13}$ Richet evidently, as was pointed out above, experimented with animals whose vagi had already been exhausted, so that section of them or atropinization could not make any difference.

The vagi of dogs vary considerably in their relative excitability and endurance. This constitutes the important personal factor in dogs.

Vagus inhibition is, in dogs, the great factor in the causation of sudden death under chloroform.

This is in accord with the conclusions of Morat and Doyen, ${ }^{11}$ Dastre ${ }^{10}$ Hare $^{5}$ and Thornton ${ }^{9}$ (conditionally).

3.- The central medullary vasomotor system is stimulated, at any rate for a time, by chloroform.

This is in accord with the conclusions of Gaskell and Shore, ${ }^{12}$ and Dastre. ${ }^{10}$ It is opposed to the views of every one else.

Oncometer experiments show that the net result of chloroform on the vessels of the spleen, bowel, and kidney shortly after the commencement of inhalation is dilatation. The effects of chloroform on the peripheral mechanism is paralytic, and on the central vasomotor system is stimulative, so that the effect upon the intact animal is some algebraic sum of the increased stimulus reaching the muscle cells, and of their diminished power to respond. At the very beginning of inhalation the second factor may predominate.

Gaskell and Shore ${ }^{12}$ found that when chloroform was delivered to the brain alone vascular constriction ensued, and they concluded that chloroform did not paralyse the vasomotor centres. Hill, ${ }^{1}$ on the other hand, found that when 
chloroform was delivered to the intact animal vascular relaxation ensued, and inferred that vasomotor paralysis occasioned it. My experiments demonstrate that the results of both of these investigations are correct. They further show that Hill's results were from peripheral and not central paralysis, and that the amount of vascular relaxation that Hill demonstrated was the resultant of these two opposing factors. There appears nothing to justify the assumption of Dastre ${ }^{10}$ that vascular constriction is a result of the administration of chloroform.

The cause of the fall of blood pressure from the administration of chloroform is paralysis of the muscle cells of the heart and of the arterioles, the fall may be further augmented by slowing of the heart's rate.

4.-Failure of respiration in inhalation experiments is mainly due to fall in blood pressure. With a good blood pressure, failure of respiration by inhalation of chloroform is practically impossible.

Restoration of respiration is dependent upon restoration of blood pressure.

These conclusions are in accord with those of Gaskell and Shore $^{12}$ and Hill. ${ }^{1}$ They are opposed to those of the Hyderabad Commission.

Failure of respiration occurring in the induction or early period of anaesthesia happens as frequently aiter as it does before the heart stops.

This is in accord with the observations of Wood, ${ }^{6}$ Snow $^{3}$ the Glasgow Committee, ${ }^{5}$ MacWilliam, ${ }^{7}$ Gaskell and Shore, ${ }^{12}$ Dastre, ${ }^{10}$ Morat and Doyen, ${ }^{11}$ Richet, ${ }^{13}$ Hill, ${ }^{1}$ Duplay and Hallion. ${ }^{16}$ It is opposed to the conclusions of the Hyderabad Commission ${ }^{y}$ and Hare and Thornton. ${ }^{9}$ Since sudden circulatory stoppage is due to the vagi inhibiting the heart, it is readily perceived that this may happen before the respiratory mechanism fails, as Tracings 8 and 9 show. In consequence of the Hyderabad Commission having taken no records of the period of induction or early anaesthesia, they obtained no instances of sudden heart stoppage from chloroform alone. For the same reason they never got an instance of heart stoppage before the respiration failed.

I have emphasized above (1) that the chances of dangerous vagus inhibition in chloroform administration, are greatly increased by imperfect respiration; (2) that respiration fails when the blood pressure falls greatly trom cardiac inhibition or other causes.

In these two series of phenomena we have material for the formation of a vicious circle, for example, supposing (as happened in some cases) that from the inhalation of too strong vapour of chloroform cardiac inhibition occurs, this occasions (I) dangerous fall of blood pressure; (2) cessation of respiration, and the asphyxia consequent thereupon itself increases the inhibition. Or supposing (as happened in some other cases) the administration of too strong vapour occasions by its direct action upon cardiac muscle a rapid fall of blood pressure. This is followed by cessation of respiration, and the ensuing asphyxia is very liable to produce sudden and often fatal inhibition of the heart, which of course still further depresses the blood pressure.

I do not wish to discuss the practical application of these results at present, but one obvious moral is, use only weak vapour of chloroform (less than I per cent.) in the early stages, until the initial increased excitability of the vagus mechanism has given place to diminished excitability; in other words, take time in putting the patient under.

In conclusion, most gratefully I acknowledge my indebtedness to Professor C. J. Martin for his valuable advice and assistance throughout the course of this investigation ; also for the use of his laboratory and his generous aid in the devising of the various new pieces of apparatus required in the course of the work.

1 BRITISH MEDICAL JO

JoURAL, April it th, 1897, p. gfo. 2 Lancet, January 17th, rooo. 3 Snow, On Anaesihetics. 4 Ringer, Practitioner, vol. xxvi. p. 436. 5 BRITISH MEDICAL JOURNAL. 1880, vol. ji, p. 970. 6 Wood, ibid. August i6th, 1890, $p$ 385. 7 Mac William, ibid.. October, $1890, p$. 949. and Journal of Physiology. vol. xxv, No. 4. ${ }^{8}$ Report of the Huderubud Commission. Hare and Thornton Lancet. 1893, $\mathrm{n}$.997. 10 Dastre, Les Anesthésiques. Paris, 189o. 11 Morat and Doyen, Traite de Physiologie, vol. i, p. xo8. 12 raskell and shore, BRITISH MEDICAL JOURNAL, 1893, pp. rc6 and 17x. 13 Richet. "Anesthésie," Dictionnaire de Physiologie, $1895 . \quad 14$ Richet, Archives de Physiologie, IS04. p. 653. T5 Waller, BRTrISH M EDICAL JOU RNAL,

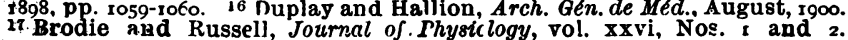

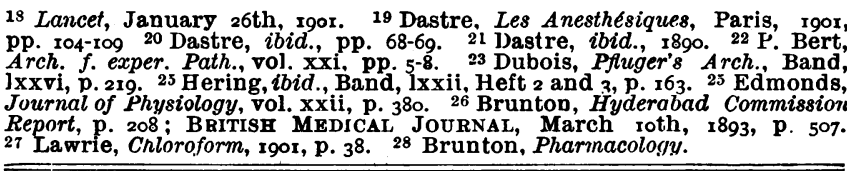

MEM ORANDA:

MEDICAL, SURGICAL，OBSTETRICAL，THERAPEUTICAL，PATHOLOGICAL，ETC.

THE SETON IN MIGRAINE.

I HAVE read with interest the article bearing on the use of the seton in obstinate cases of migraine, and can endorse the opinion on the value of the treatment. Some three months ago I had a chronic and obstinate case of migraine under treatment, a case where the pain had persisted in spite of all the drugs that had been used, and they had been many and various.

The patient was a peon in the Forest Service, and had been given six months leave on account of migraine. When he came under my care he was in a very reduced state of health on account of the almost constant and severe pain which he suffered and from want of sleep. He had also lost all appetite, and was very thin and weak. After the introduction of a seton in the back of his neck improvement was most marked and decided, and in three weeks he was almost well, had lost all pain, was able to sleep at night, his appetite had returned, and he was a different man. The seton was then removed; he was kept under treatment for about ten days afterwards, and was then returned to duty perfectly cured. He has come back to report once since then, and at that time had had no return of the old pain, and was looking in excellent health. This is the only case of the sort I have had, but is, I think, worth recording in the hope that others with more opportunities may give the treatment a trial in those obstinate cases of migraine which one gets occasionally.

Palamcottah. $\quad$ T. E. WATson, M.B., Captain I.M.S.

THE TREATMENT OF CHOREA BY LARGE DOSES OF FOWLER'S SOLUTION.

Dr. Murray, of Newcastle-upon-Tyne, speaks highly of large doses of liquor arsenicalis in the treatment of chorea, and the following case may be cited as bearing out the correctness of his conclusions.

A boy, aged 9 years, of nervous disposition and rheumatic family history, was sent home from school hy the teacher "because he could not sit quiet with the St. Vitus's dance." For eight days afterwards he attended the out-patient department of one local hospital, and for fifteen days at another, without much benefit, according to the mother's account.

When I saw him at the end of this time his movements were almost violent as regards the arms and head. He was at once put on 12-minim doses of liq. arsenicalis in sodium bicarbonate and syrup with his meals. He had a good appetite, and vomited only once on the third day. On the fifth day the eyes were affected, and there were slight cramps in the bowels, but the choreic movements were abating rapidly. "He could now feed himself without spilling, he did not knock his head against the furniture, and could now be left alone in the house." The dose of arsenic was then reduced to 5 minims, and discontinued on the eighth day. There were no further toxic signs, and the symptoms of chorea had entirely gone. The boy has now returned to school.

Bootle. ROBERT TURNER. M.A., M.D.Aberd., F.R.C.S.Edin.

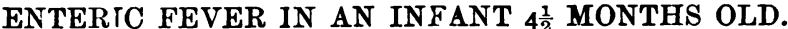
ON December Ioth, Igor, I was called to see a Dutch girl aged I years, and found her to be suffering from enteric fever at about the tenth day. She was then suckling a male child aged $4 \frac{1}{2}$ months; the child looked strong and healthy at that time. It was weaned at once, and I did not see it again till fourteen days later, when it looked extremely ill, had a temperature of $103^{\circ}$ a pulse of 112 , very soft and compressible. There was a systolic murmur over the mitral area; the spleen 